\title{
Simulations of Disordered Proteins and Systems with Conformational Heterogeneity
}

\author{
Zachary A. Levine ${ }^{a, b}$ and Joan-Emma Shea ${ }^{a, b^{*}}$
}

a) Department of Chemistry and Biochemistry,
University of California Santa Barbara, Santa Barbara, CA 93106, USA
b) Department of Physics,
University of California Santa Barbara, Santa Barbara, CA 93106, USA

Keywords: Intrinsically Disordered Proteins, Intrinsically Disordered Regions, Atomistic Simulations, Molecular Dynamics, Force Fields, Aggregation, Self-Assembly

Short Title: Simulations of Disordered Proteins

*Corresponding author:

Joan-Emma Shea

Department of Chemistry and Biochemistry, University of California Santa Barbara, Santa Barbara, CA 93106

Tel: 1 (805) 893-5604

Email: shea@chem.ucsb.edu 


\begin{abstract}
Intrinsically disordered proteins and protein regions can facilitate a wide variety of complex physiological processes such as binding, signaling, and formation of membraneless organelles. They can however also play pathological roles by aggregating into cytotoxic oligomers and fibrils. Characterizing the structure and function of disordered proteins is an onerous task, primarily because these proteins adopt transient structures, which are difficult to capture in experiments. Simulations have emerged as a powerful tool for interpreting and augmenting experimental measurements of IDPs. In this review we focus on computer simulations of disordered protein structures, functions, assemblies, and emerging questions that, taken together, give an overview of the field as it exists today.
\end{abstract}

\title{
Introduction
}

A longstanding guiding principle in molecular biology is that the amino acid sequence of a protein determines its three dimensional fold, and that structure is inherently linked to function. However, nearly a third of proteins encoded in the human proteome contain intrinsically disordered regions (IDRs) and lack a discernible tertiary structure. ${ }^{1}$ Intrinsically disordered proteins (IDPs), which are made up entirely of IDRs, can take on a multitude of disparate conformations, adopt transient secondary structures, and carry out multiple functions, challenging the classical structure-function paradigm. Historically, IDRs were thought of as flexible linkers between more ordered protein regions, but more recent studies have revealed that IDRs can enhance protein function, interact with a large number of proteins and protein ligands, and allow for a large number of post-translational modifications (PTMs) to take place. ${ }^{2,3}$ IDRs can also contribute to the regulation of protein function by enabling transient exposure of small amino acid groups to water and other molecules, thereby balancing dichotomous protein behaviors ${ }^{3,4}$ and populating highly heterogeneous conformations that rapidly interconvert. ${ }^{1}$ Given the immense complexity of IDRs and IDPs, a number of functions and transient structures have been identified by Van der Lee et al. ${ }^{3}$ based on 150 unique IDRs that, taken together, help describe the molecular basis for intrinsic protein behavior. Structural subtypes, functional features (smaller motifs embedded in IDRs), macroscopic structures, sequence-specific behaviors, protein interactions, evolutions, regulatory behaviors, and biophysical properties have been studied in detail, ${ }^{1,3}$ most of which are catalogued in publically accessible databases. ${ }^{5-9}$

IDPs typically contain a larger number of polar and charged amino acids, and fewer hydrophobic amino acids compared to globular proteins, thereby hampering the formation of stable hydrophobic cores. ${ }^{10}$ Transient secondary and tertiary structures can arise as a result of interactions between charged groups and due to the fact that water is a poor solvent for polypeptide chains. Metrics such as the fraction of positive-to-negative residues can be used to quantify protein transitions from globular to intrinsically disordered, ${ }^{11}$ and to classify IDPs into three main categories: polar tracts, polyampholytes, and polyelectrolytes, as shown in Figure 1. 

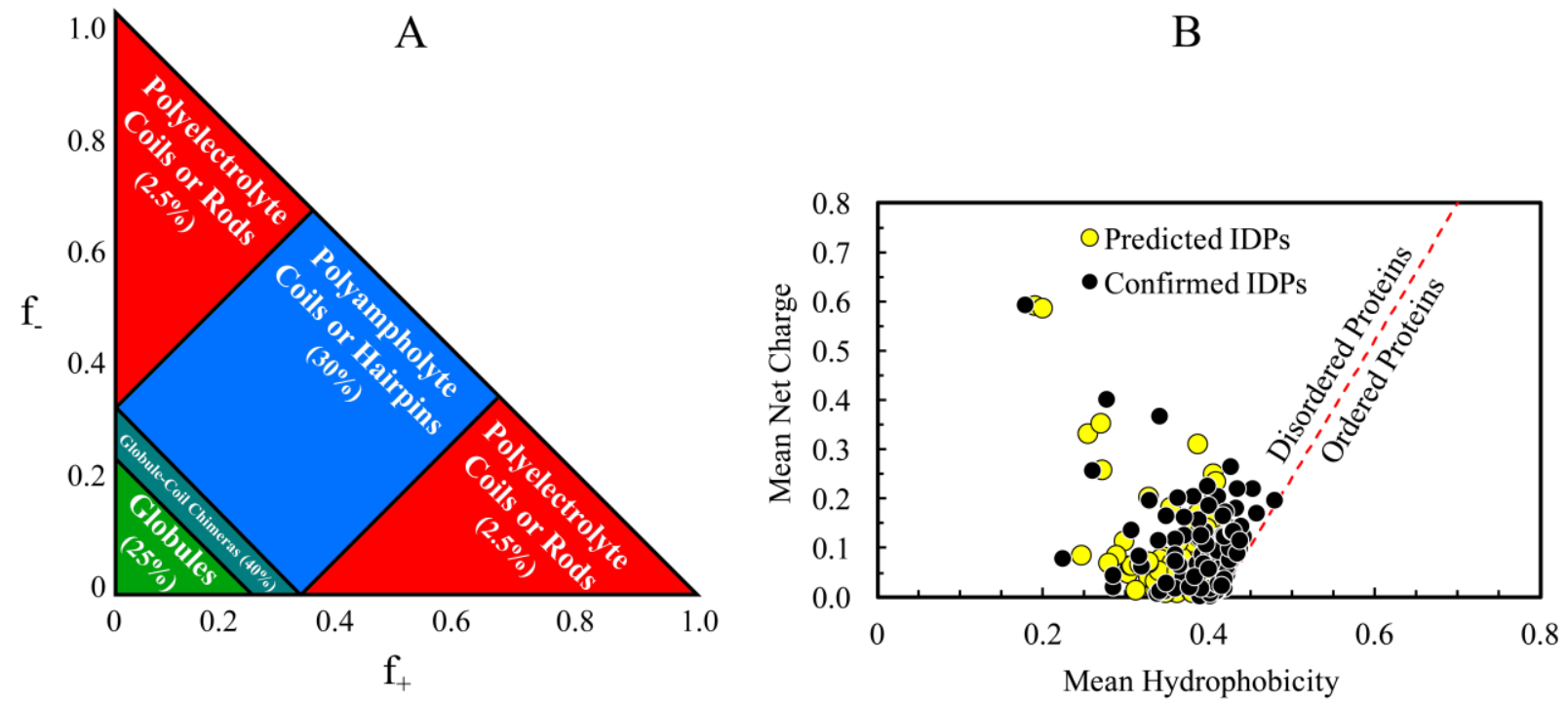

Figure 1. [A] IDPs can be categorized by the amount of positively charged $\left(f_{+}\right)$and negatively charged ( $f_{-}$) amino acids they contain, which can help predict their behaviors. [B] A majority of disordered proteins tend to exhibit relatively high net charges and very little hydrophobicity. Figure adapted from reference 3 .

The IDR/IDP field is an active area of research that has benefited from a close collaboration between experimental and computational studies. Common experimental techniques used to characterize IDPs involve using SAXS (small angle X-ray scattering) and FRET (Förster resonance energy transfer) measurements to extract chain dimensions, or NMR (nuclear magnetic resonance) for information regarding secondary structure. However, characterizing transient conformational ensembles using ensemble averaging methods has several limitations. A number of computational tools that incorporate machine-learning predictors based on known interaction energies, sequence information, and experimental constraints, can be utilized alongside experiment. Researchers are also increasingly turning to atomistic ab-initio simulations to evaluate IDP structures and functions. In this article we will review recent simulations of IDRs and IDPs, emphasize their strengths and limitations, and discuss how simulations have contributed to our present understanding of IDP structure and function.

\section{Computational Modeling of IDP structures}

Computational modeling is becomingly an increasingly attractive complement to experimental studies of IDPs, for which characterization of transient structures pose challenges.

Computational platforms that study IDP structure are often initially informed from prior experiments, and include methodologies that combine SAXS and NMR data with computational methods to characterize IDP ensembles. ${ }^{12}$ Knowledge-based approaches such as TraDES $^{13}$ and Flexible-Meccano ${ }^{13,14}$ can be used initially to generate IDP ensembles containing random-coil conformations. Then, back calculations of experimental NMR observables, augmented with 
Bayesian weighting protocols ${ }^{15,16}$ are used to narrow the initial pool of structures to a set that best matches with NMR chemical shifts, ${ }^{12}$ and residual dipolar coupling data. ${ }^{17}$ Examples of these methods include the genetic algorithm-based ASTEROIDS program, ${ }^{14}$ energy-weighted methods, ${ }^{18}$ and the ENSEMBLE program. ${ }^{19}$ It should be kept in mind that resulting IDP ensembles are inherently under-determined since the number of degrees of freedom far exceeds the number of experimental constraints. Improvements in back-calculation algorithms and in the structural databases from which back-calculations are applied to are ongoing areas of research.

Emerging alternatives to knowledge-based approaches for IDP structure determination include de-novo approaches, which differ from the former in that they combine empirical force fields with molecular dynamics or Monte Carlo simulations. As with knowledge-based methods, denovo structures are validated by back-calculations to experimental observables. However, a net advantage is that these methods generate Boltzmann-weighted ensembles and other dynamic metrics that can be correlated to NMR measurements. Head-Gordon and co-workers have shown that the ensembles generated by knowledge-based approaches are qualitatively different from those generated by ab-initio methods, especially with respect to chain extension and secondary structure. ${ }^{20}$

In the case of de-novo methods, two critical issues need to be considered. The first is the accuracy of the force fields that are used, while the second is the quality of the sampling. Enhanced sampling protocols are required to obtain adequate sampling of an IDP's conformational landscape. This is especially important because conformational changes to IDPs can occur on timescales up to milliseconds, which are not amenable to conventional simulations. Protocols such as Replica-Exchange Molecular Dynamics (REMD) ${ }^{21}$ and Metadynamics ${ }^{22-24}$ have been particularly successful in this regard, and have been used to characterize the monomeric structures of multiple IDPs. Force field optimization remains a challenge and an active field of research. In classical molecular force fields, ${ }^{25}$ quantum information and chemical effects (including electronic polarization, the forming and breaking of bonds, etc.) must be mapped onto classical parameters and a simple Hamiltonian containing Coulombic interactions, van der Waals interactions, rigid bonds, and angular and dihedral terms. Furthermore, many protein partial charges are derived from simulations in the gas phase, and protein force fields are coupled with equally simplistic water models, ${ }^{26}$ many of which fail to reproduce basic experimental properties like diffusion or phase-transitions at the proper temperatures. ${ }^{27}$ While many existing protein and water models are adequate for room temperature simulations of globular proteins, where hydrophobic residues are often shielded from water near the protein core, simulations of extended IDPs require increased solvation accuracy since exposure to water is commonplace. Existing protein force fields such as GROMOS, ${ }^{28} \mathrm{OPLS}_{\mathrm{AAA}},{ }^{29}$ and $\mathrm{CHARMM}{ }^{26}$ continue to be parameterized with older TIP3P, ${ }^{30} \mathrm{SPC}^{31}$ or $\mathrm{SPC} / \mathrm{E}^{32}$ water models. Many of these force fields result in IDPs that are too compact, ${ }^{33}$ too hydrophobic, ${ }^{34}$ and which favor larger protein-protein interactions ${ }^{35}$ over experiment.

Initial attempts to correct these errors by force field re-parameterization resulted in more accurate solvation free energies, however globular proteins exhibited spurious instabilities as a 
result. ${ }^{36}$ Best and Mittal ${ }^{37}$ advocated for fine-tuning or tweaking existing protein backbones in the presence of modern water models, since errors in backbone parameters have a cumulative effect applied to every residue in the system. They proposed an update to the AMBER03* force field (termed AMBER03w) ${ }^{37}$ that utilizes the updated TIP4P/2005 water model ${ }^{38}$ along with corrections to protein backbone dihedrals, allowing for improved IDP dynamics and helical propensities to be sampled in MD simulations. Increased radii of gyrations for non-helical IDPs are observed in AMBER03w (consistent with experiment) as compared to the AMBER03* force field with TIP3P water, due to more favorable enthalpic peptide-solvent interactions. Using the AMBER03w force field, Mittal and co-workers showed that IDPs with polyelectrolyte (such as PROT-C) and polyampholyte (PROT-N) characteristics followed the scaling laws predicted by polymer theory, and that the radii of gyration extracted from simulation followed the same temperature dependent trends as those obtained from FRET measurements. ${ }^{39}$

Several comparative studies of IDPs under multiple force fields have recently been published. For example, simulations by the Garcia group considered a range of force fields and water models using replica exchange molecular dynamics, to characterize the conformations sampled by the amyloid- $\beta$ peptide and related mutants linked to early-onset Alzheimer's Disease. ${ }^{40}$ The protein-water force field pairs included OPLS-AA/L and TIP3P, AMBER99sb-ILDN and TIP4P-Ew, and CHARMM22* and a CHARMM-modified TIP3P (TIP3SP). While the overall tertiary structures of the peptide were similar in each model, with comparable compact maps generated for each force field, CHARMM22* populated more extended conformations than the other force fields while OPLS-AA/L yielded the most compact structures. Peptide secondary structures did vary between force fields, particularly in the hydrophobic core region. Since this region is critical for aggregation, and adjacent to sites that are linked with familial mutations, errors introduced in secondary structure at the monomeric level may translate into significant differences in larger oligomers. All three force fields adequately captured NMR data such as J-couplings, however, Head-Gordon and co-workers note that some NMR data such as J-couplings cannot always distinguish between structural ensembles of IDPs that are qualitatively different. ${ }^{41}$

Capturing the right balance between protein-water interactions appears to be key to correctly describing IDP dimensions. Best and co-workers subsequently applied modifications to shortrange protein-water interactions in $A M B E R 03 w$ by increasing their Lennard-Jones interactions by $10 \%$ (compared to other coupling factors), resulting in more realistic IDP dimensions (compared to SAXS and FRET). ${ }^{42}$ This force field, named AMBER03ws (water-scaled), resulted in qualitatively better solvation free energies for each amino acid, and exhibited only modest effects on $\alpha$-helix and $\beta$-strand forming peptides, making this force field suitable for the study of both globular proteins and IDPs. Figure 2 shows a comparison of the radius of gyration of an IDP derived from the mussel-foot protein, simulated using replica exchange molecular dynamics simulations in both the AMBER03*-TIP3P and AMBER03ws-TIP4P/2005 force fields. The IDP simulated in AMBER03* adopts collapsed conformations, in contrast to the more extended ones populated in the AMBERws force field.

An alternate approach to addressing the water-protein interactions of IDPs comes in the form of the TIP4P-D water force-field by Piana and co-workers. ${ }^{43}$ This modified water model seeks to 
account for the fact that existing water models underestimate dispersion forces. The correction was implemented by increasing the Lennard-Jones $C_{6}$ parameter by $50 \%$ (in contrast to AMBER03ws that scales both the $C_{6}$ and $C_{12}$ terms by $10 \%$ ). This modification has, in some instances, led to improvements. While AMBER99sb-ILDN with TIP3P yielded overly collapsed IDP structures, AMBER99sb-ILDN ${ }^{44}$ with TIP4P-D yielded comparable results to AMBER03ws with regards to chain dimensions. ${ }^{45}$ Interestingly, the combination of TIP4P-D with CHARMM22* yielded structures that were overly expanded compared to experiment, while the CHARMM22* force field with CHARMM-modified TIP3P water outperformed AMBER03ws in reproducing SAXS and NMR measurements of IDPs over microsecond timescales (with regards to IDP radius of gyration, hydrodynamic radius, chemical shifts, and J-couplings). ${ }^{46}$ This illustrates the many challenges of "mixing, matching and tweaking" water models to work with existing force fields. An alternate and promising approach to force field parameterization involves using Kirkwood-Buff theories of solvation, as recently implemented by Gräter and coworkers. $^{47}$

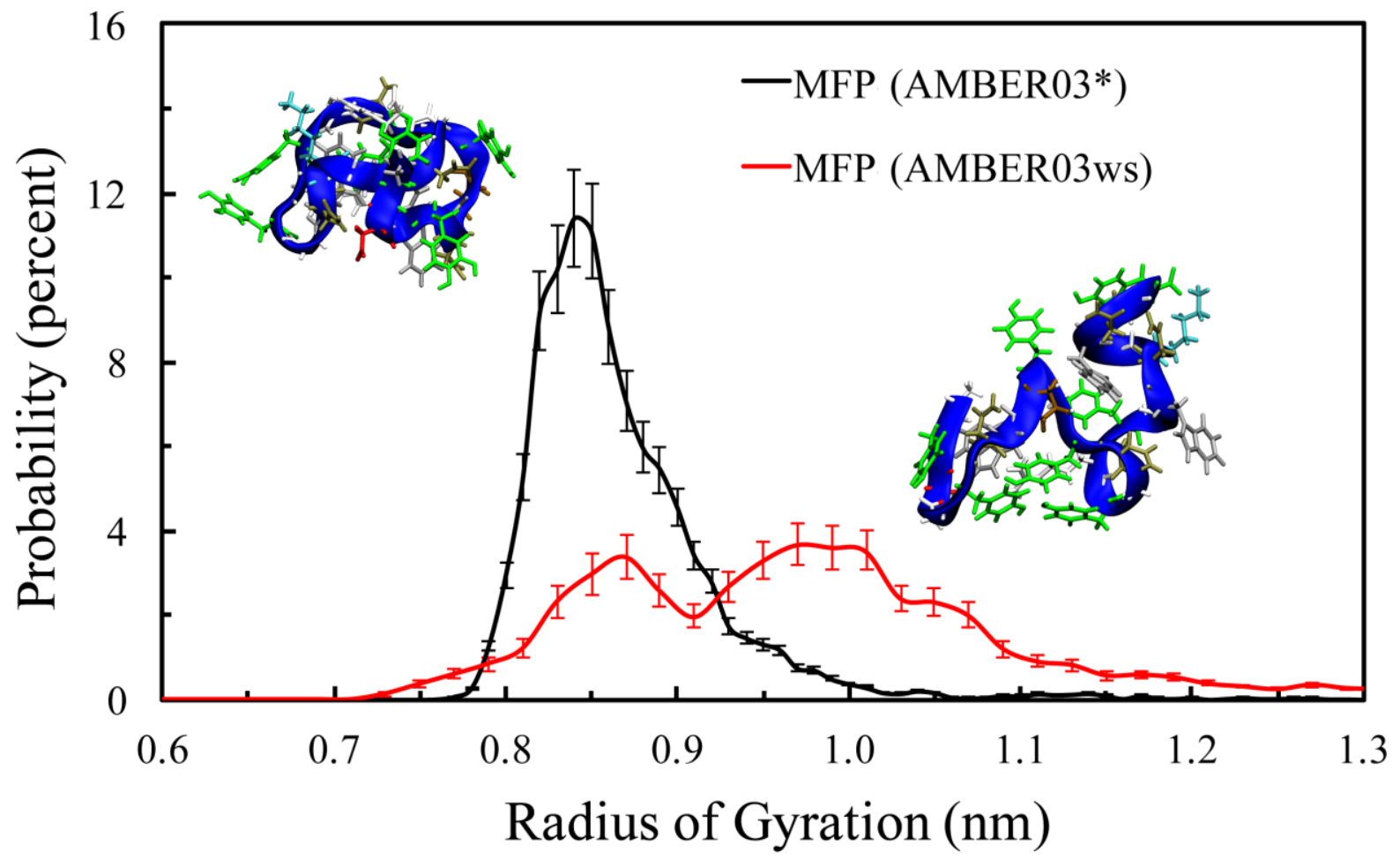

Figure 2. Radii of gyration for intrinsically disordered mussel-foot proteins (MFPs) vary considerably between AMBER03* (black) and AMBER03ws (red) force fields, with the AMBER03* force field leading to a higher population of compact structures. Snapshots from simulation show compact and extended conformations. Adapted from ${ }^{48}$. 


\section{Computational Modeling of IDP Functions}

In addition to characterizing the structure of monomeric IDPs, MD simulations have also yielded important insight on how IDPs bind to other proteins, ${ }^{47,49}$ a process that typically imparts structure to the IDP. ${ }^{1,3,50}$ Coarse-grained models have enjoyed some success in describing coupled binding and folding. The significant advantage of these models is their reduced computational cost compared to atomistic simulations. The simplest coarse-grained models are Go-models, which are structure based-models that represent amino acids as identical beads on a chain, where only native interactions are favored to drive assembly. The use of these models is limited to systems where the structures of the bound complexes are known. Improvements to Go-models include hybrid Go-models that incorporate amino acid-specific interactions and non-native interactions. ${ }^{51}$ When applied to, e.g. a pKID-KIX system, improved Go-models showed higher binding rates compared to standard models via stabilization of the encounter complexes. 52 Incorporation of charged amino acids in Go-models through Coulombic interactions revealed an important role for electrostatic interactions in promoting coupled binding and folding. ${ }^{53}$ Coupled-binding and -folding has also been studied using enhanced sampling simulations with atomistic descriptions of the proteins under both implicit and explicit solvent. These simulations are even more challenging than simply characterizing the monomeric structures of IDPs, as they involve not one, but two proteins to adequately sample.

There have also been suggestions that the transient IDP secondary structures encountered in bulk solution are representative of the structures that must occur for binding and signaling to take place (conformational selection mechanism), ${ }^{12}$ however others have suggested that IDPs bind in a disordered state, adopting structure only when bound (induced fit mechanism). ${ }^{54}$ Coarse-grained and atomistic simulations have shown evidence for both mechanisms. Implicit solvent atomistic simulations by Chen have shown that the C-terminus of the tumor suppressor p53 protein (residues 367-392) adopts folded conformations prior to binding, but interestingly, these folded conformations needed to unfold before binding could occur. ${ }^{50}$ In explicit solvent atomistic simulations, Higo and co-workers found that the IDP NRSF (neural restrictive silencer factor) populates a range of structured conformations in its unbound state as well as multiple bound native structures. ${ }^{55}$ These simulations suggest a hybrid mechanism that was termed the 'extended conformational selection mechanism' that encompassed both elements of the conformational selection mechanism and the induced fit mechanism cooperatively.

The analytically proposed "fly-casting" model ${ }^{54}$ postulates that there is a kinetic advantage for IDPs to bind compared to folded proteins, with the unstructured protein chain binding to the target and then folding while bound, with the target protein "pulling" the IDP in. This mechanism would allow IDPs to adopt multiple unique conformations and functions upon binding, a property known as moonlighting. ${ }^{1}$ The kinetic advantage of fly-casting was initially believed to be linked to the larger capture radii of extended IDPs, however this would lead to slower diffusion rates. ${ }^{56}$ Go-model simulations, augmented with non-native interactions and electrostatic interactions, suggested instead that these increased binding rates were due to the conformational flexibility of IDPs and to electrostatic steering, thereby refining the fly-casting model. ${ }^{57}$ 


\section{Computational Modeling of IDP Assemblies: Liquid Droplets and Amyloid Fibrils}

With their lack of a native folded structure, IDPs are prone to self-assembly, particularly under the crowded conditions found in cells. In some instances this can lead to the accumulation of pathological assemblies that are cytotoxic. A hallmark of this assembly process is the formation of amyloid fibrils, extended structures with a characteristic cross- $\beta$ arrangement of strands. Examples of amyloid-related diseases involving IDPs include Parkinson's Disease ( $\alpha$-synuclein protein), Alzheimer's Disease (amyloid- $\beta$ ? (?]?]? Amylin peptide). Simulations at both the coarse-grained and atomistic levels have helped elucidate the aggregation mechanisms of IDPs. This topic has been extensively covered in recent review articles, therefore only limited examples will be provided here. ${ }^{58,59}$ The aggregation process is common to many different IDP sequences and as such lends itself to the use of coarse-grained models which focus on generic (polymer) characteristics of the polypeptide chain rather than on the precise chemical details of individual amino acids. Coarsegrained models are attractive because they can easily accommodate a large number of peptides and longer timescales as compared to atomistic models. Off-lattice models (with one or more beads per amino acid) have been extremely successful in mapping the aggregation process from monomers and oligomers to fibrils, and in capturing the nucleation-growth mechanisms of aggregation. ${ }^{58,6061}$ Coarse-grained models have shown, for example, that fibril formation can occur directly from aggregating monomers via ordered $\beta$-rich oligomers, or from disordered aggregates that must rearrange their structures to form extended- $\beta$ fibrils. ${ }^{62}{ }^{63}$ Atomistic simulations have characterized the early stages of aggregation using enhanced sampling methods such as replica exchange molecular dynamics and metadynamics, by focusing on monomers (described earlier), dimers, and in the case of small peptides, slightly larger oligomers. ${ }^{64}$ They have also provided significant insights into the interaction of IDPs with lipid bilayers ${ }^{65}$, and into the role of co-solvents in modulating IDP conformation. ${ }^{4}{ }^{66}$ Atomistic simulations have also characterized the final stages of aggregation by focusing on models of amyloid fibrils, ${ }^{67}$ on fibrils growth mechanisms (including the dock-lock mechanism ${ }^{68}{ }^{69}$ ), and studied the interactions between small molecules or peptide inhibitors with fibrils. Simulations of amyloid-dyes (such as Congo Red or Thioflavin T, which are used experimentally to identify fibrils) have yielded important mechanistic insight into the binding modes of these molecules, and suggested ways to generate improved dyes. ${ }^{70}$

In addition to forming amyloid-fibrils, IDPs can also phase separate into a protein-rich phase in equilibrium with a protein-depleted bulk phase, via either simple or complex coacervation. ${ }^{71}$ Simple coacervation involves phase separation of a single protein species, and occurs primarily with IDPs containing few charged amino acids. Complex coacervation occurs in IDPs that typically contain one type of charge (disfavoring self-association), where a complementary protein or nucleic acid of opposing charge is needed for phase separation to occur. In contrast to the process of amyloid formation, phase separation of IDPs into liquid droplets is believed to facilitate beneficial physiological roles by forming membrane-less organelles for storage or delivery of concentrated peptides. These organelles include Cajal bodies, nucleoli, and stress 
granules, many of which contribute to signal transduction, gene regulation, and ribosomal assembly. ${ }^{1,72}$ Under certain conditions (mutations, post-translational modifications, changes in the environment) IDP droplets can convert into amyloids (Figure 3). While still somewhat of a novelty in the IDP world, the study of coacervation dates back nearly a century, with the term first coined in the context of colloidal solutions in $1929 .{ }^{73}$ Coacervate formation has been observed in both electrolyte and non-electrolyte solutions, and simple analytical theories have been developed in this context, however their ability to predict coacervation has been limited to a small region of parameter space. ${ }^{74,75}{ }^{76}$ From a computational standpoint, coacervation studies are still in their infancy. These systems are difficult to study in detail because they require coarse-grained descriptions in order to reasonably model the large number of particles involved in assembly, but at the same time, these simplified models must capture the delicate interplay of electrostatic interactions, hydrophobic interactions, salt screening, and conformational entropy of the polymer chain. While a mean field approximation on its own has been shown to be deficient in describing coacervation, models that go beyond mean field descriptions have had some success, including the use of Field Theoretic Simulations (FTS) of polyelectrolyte coacervation. ${ }^{77}$ With regards to simulations of biologically relevant IDPs, the literature is still thin. Monte Carlo atomistic simulations using the ABSINTH implicit solvent model $^{78}$ have been used by Pak et al. with experimental techniques such as in-vitro cellular microscopy, to study the coacervation of the intracellular domain of Nephrin (NICD). ${ }^{72}$ While such simulations cannot easily model the formation of droplets (due to inadequate sampling), they can nonetheless provide invaluable insights into the interactions that drive and stabilize coacervation. Coupled with experiments, these simulations showed that the primary factor determining phase separation was overall amino acid composition, not the precise sequence of NICD. Coacervation was primarily promoted by large, negatively charged IDRs and a combination of aromatic (Tyr and Trp) and hydrophobic (Met and Leu) residues distributed across NICD. 


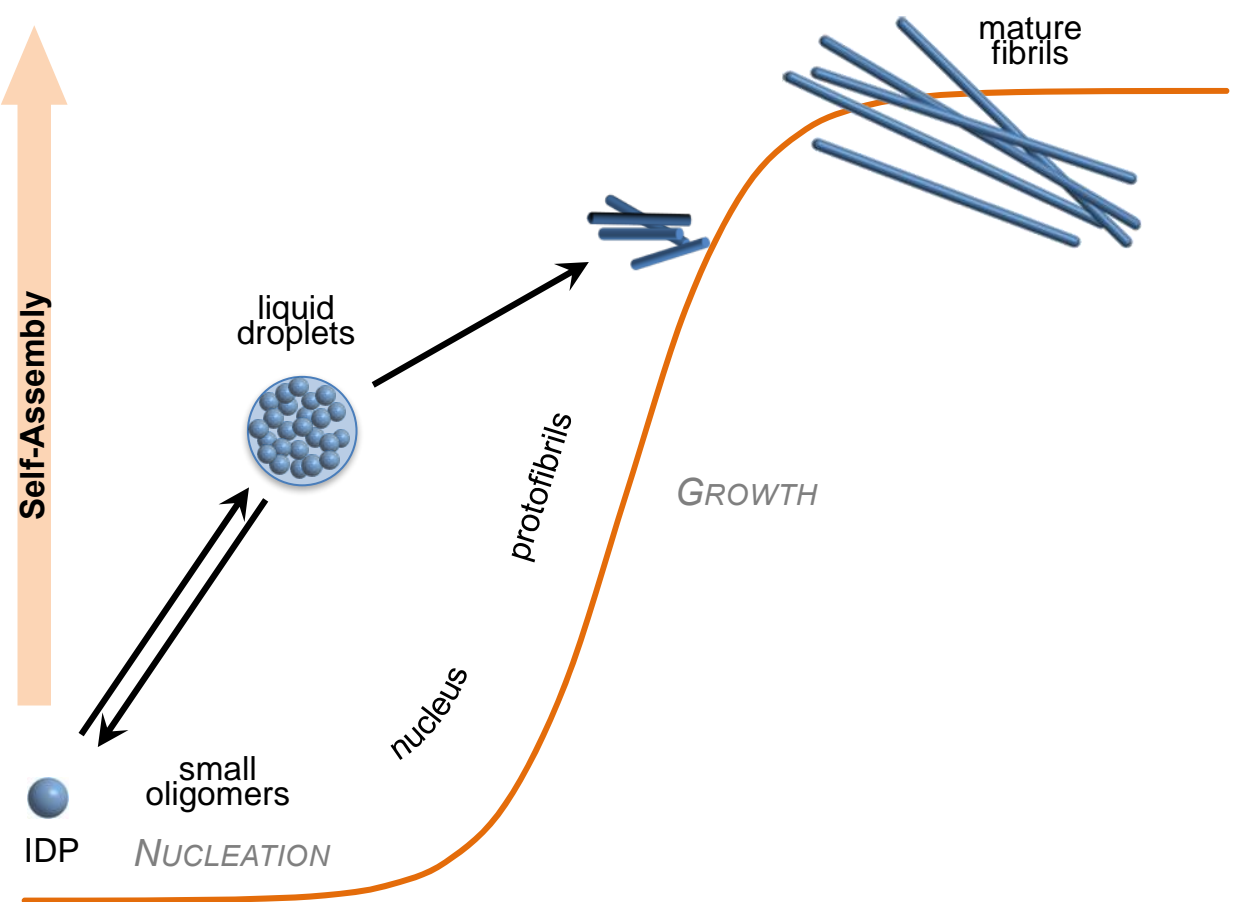

Time

Figure 3. Pathways to IDP fibril formation include formation of liquid droplets (coacervates), in addition to the conventional nucleation-growth pathway.

\section{Future Directions in IDP Modeling}

While simulations of IDPs provide an important tool to help interpret experimental observations, a number of unresolved issues still need to be addressed. For instance, the question of whether it is better to minimally tweak existing IDP force fields using updated solvent models, versus reparametrizing entire force fields from scratch is unclear. Modifying pieces of an existing force field, especially only the van der Waals components between solute and solvent, can have unforeseen consequences if different solvents are utilized, especially if large concentrations of co-solvents or counter ions are used in addition to water. Similarly, utilization of a single global variable to scale all amino acid-water interactions is somewhat crude, and could be improved by implementing multiple coupling factors customized to each amino acid-solvent pair, though such a task is not far off from constructing an entirely new force field. One major benefit, however, to fine-tuning existing IDP force fields is that updated coupling parameters can be transferred to analogous force fields. ${ }^{42}$ Improvements in force field accuracy, sampling, analysis, and in the methodologies used to compare experimental observables and validate computational models, are much needed. 
The field of droplet formation is wide open from a computational perspective, and there is ample room to make an impact in understanding the mechanisms of droplet formation, and the transition between droplets and amyloid fibrils. Another burgeoning field is the study of crowding molecules on IDP conformation and aggregation, and how cellular environments can modulate the structures and functions of IDPs. 61798081

\section{Acknowledgements}

Partial financial support was provided by NSF grant MCB-1158577 and by the MRSEC Program of the National Science Foundation under Award No. DMR 1121053. This work used the Extreme Science and Engineering Discovery Environment - XSEDE (TG-MCA05S027), which is supported by National Science Foundation grant number ACl-1053575. The authors also acknowledge the Texas Advanced Computing Center (TACC) at The University of Texas at Austin for providing HPC resources that have contributed to the research results reported within this paper. We acknowledge support from the Center for Scientific Computing from the CNSI, MRL: an NSF MRSEC (DMR-1121053) and NSF CNS-0960316. We thank G. Zerze and J. Mittal for contributing to Figure 2.

\section{REFERENCES}

1 Csizmok, V., Follis, A. V., Kriwacki, R. W. \& Forman-Kay, J. D. Dynamic Protein Interaction Networks and New Structural Paradigms in Signaling. Chem Rev 116, 6424-6462, doi:10.1021/acs.chemrev.5b00548 (2016).

2 Bah, A. \& Forman-Kay, J. D. Modulation of Intrinsically Disordered Protein Function by Post-translational Modifications. J Biol Chem 291, 6696-6705, doi:10.1074/jbc.R115.695056 (2016).

3 van der Lee, R. et al. Classification of intrinsically disordered regions and proteins. Chem Rev 114, 6589-6631, doi:10.1021/cr400525m (2014).

4 Levine, Z. A., Larini, L., LaPointe, N. E., Feinstein, S. C. \& Shea, J.-E. Regulation and aggregation of intrinsically disordered peptides. Proceedings of the National Academy of Sciences 112, 2758-2763, doi:10.1073/pnas.1418155112 (2015).

5 Sickmeier, M. et al. DisProt: the database of disordered proteins. Nucleic acids research 35, D786-D793 (2007).

6 Fukuchi, S. et al. IDEAL in 2014 illustrates interaction networks composed of intrinsically disordered proteins and their binding partners. Nucleic acids research 42, D320-D325 (2014).

7 Rose, P. W. et al. The RCSB Protein Data Bank: new resources for research and education. Nucleic acids research 41, D475-D482 (2013).

8 Di Domenico, T., Walsh, I., Martin, A. J. \& Tosatto, S. C. MobiDB: a comprehensive database of intrinsic protein disorder annotations. Bioinformatics 28, 2080-2081 (2012).

9 Oates, M. E. et al. D2P2: database of disordered protein predictions. Nucleic acids research 41, D508-D516 (2013). 
10 Uversky, V. N., Gillespie, J. R. \& Fink, A. L. Why are "natively unfolded" proteins unstructured under physiologic conditions? Proteins: structure, function, and bioinformatics 41, 415-427 (2000).

11 Das, R. K. \& Pappu, R. V. Conformations of intrinsically disordered proteins are influenced by linear sequence distributions of oppositely charged residues. Proceedings of the National Academy of Sciences 110, 13392-13397 (2013).

12 Schneider, R. et al. Visualizing the Molecular Recognition Trajectory of an Intrinsically Disordered Protein Using Multinuclear Relaxation Dispersion NMR. J Am Chem Soc 137, 1220-1229 (2015).

13 Feldman, H. J. \& Hogue, C. W. V. A fast method to sample real protein conformational space. Proteins 39, 112-131, doi:Doi 10.1002/(Sici)1097-0134(20000501)39:2<112::AidProt2>3.0.Co;2-B (2000).

14 Schneider, R. et al. Towards a robust description of intrinsic protein disorder using nuclear magnetic resonance spectroscopy. Mol Biosyst 8, 58-68, doi:10.1039/c1mb05291h (2012).

15 Fisher, C. K., Huang, A. \& Stultz, C. M. Modeling Intrinsically Disordered Proteins with Bayesian Statistics. J Am Chem Soc 132, 14919-14927, doi:10.1021/ja105832g (2010).

16 Brookes, D. H. \& Head-Gordon, T. Experimental Inferential Structure Determination of Ensembles for Intrinsically Disordered Proteins. J Am Chem Soc 138, 4530-4538, doi:10.1021/jacs.6b00351 (2016).

17 Bertoncini, C. W. et al. Release of long-range tertiary interactions potentiates aggregation of natively unstructured $\alpha$-synuclein. Proceedings of the National Academy of Sciences of the United States of America 102, 1430-1435 (2005).

18 Yoon, M.-K. et al. Residual structure within the disordered C-terminal segment of p21Waf1/Cip1/Sdi1 and its implications for molecular recognition. Protein Science 18, 337-347, doi:10.1002/pro.34 (2009).

19 Krzeminski, M., Marsh, J. A., Neale, C., Choy, W. Y. \& Forman-Kay, J. D. Characterization of disordered proteins with ENSEMBLE. Bioinformatics 29, 398-399, doi:10.1093/bioinformatics/bts701 (2013).

20 Ball, K. A., Wemmer, D. E. \& Head-Gordon, T. Comparison of Structure Determination Methods for Intrinsically Disordered Amyloid-beta Peptides. J Phys Chem B 118, 64056416, doi:10.1021/jp410275y (2014).

21 Sugita, Y. \& Okamoto, Y. Replica-exchange molecular dynamics method for protein folding. Chem Phys Lett 314, 141-151, doi:Doi 10.1016/S0009-2614(99)01123-9 (1999).

22 Levine, Z. A., Fischer, S. A., Shea, J.-E. \& Pfaendtner, J. Trp-Cage Folding on Organic Surfaces. The Journal of Physical Chemistry B 119, 10417-10425, doi:10.1021/acs.jpcb.5b04213 (2015).

23 Zerze, G. H., Miller, C. M., Granata, D. \& Mittal, J. Free Energy Surface of an Intrinsically Disordered Protein: Comparison between Temperature Replica Exchange Molecular Dynamics and Bias-Exchange Metadynamics. J Chem Theory Comput 11, 2776-2782 (2015).

24 Bonomi, M. et al. PLUMED: A portable plugin for free-energy calculations with molecular dynamics. Comput Phys Commun 180, 1961-1972 (2009). 
25 Hornak, V. et al. Comparison of multiple amber force fields and development of improved protein backbone parameters. Proteins-Structure Function and Bioinformatics 65, 712-725 (2006).

26 Huang, J. \& MacKerell, A. D. CHARMM36 all-atom additive protein force field: Validation based on comparison to NMR data. Journal of computational chemistry 34, 2135-2145 (2013).

27 Ashbaugh, H. S., Collett, N. J., Hatch, H. W. \& Staton, J. A. Assessing the thermodynamic signatures of hydrophobic hydration for several common water models. The Journal of chemical physics 132, 124504 (2010).

28 Oostenbrink, C., Villa, A., Mark, A. E. \& Van Gunsteren, W. F. A biomolecular force field based on the free enthalpy of hydration and solvation: the GROMOS force-field parameter sets 53A5 and 53A6. Journal of computational chemistry 25, 1656-1676 (2004).

29 Robertson, M. J., Tirado-Rives, J. \& Jorgensen, W. L. Improved peptide and protein torsional energetics with the OPLS-AA force field. J Chem Theory Comput 11, 3499-3509 (2015).

30 Jorgensen, W. L., Chandrasekhar, J., Madura, J. D., Impey, R. W. \& Klein, M. L. Comparison of Simple Potential Functions for Simulating Liquid Water. J Chem Phys 79, 926-935, doi:Doi 10.1063/1.445869 (1983).

31 Berendsen, H. J. C., J. P. M. Postma, W. F. van Gunsteren, and J. Hermans. Interaction models for water in relation to protein hydration. In Intermolecular Forces. B. Pullman, editor. Reidel, Dordrecht, Netherlands, 331-342 (1981).

32 Berendsen, H. J. C., Grigera, J. R. \& Straatsma, T. P. The Missing Term in Effective Pair Potentials. J Phys Chem-Us 91, 6269-6271, doi:Doi 10.1021/J100308a038 (1987).

33 Nettels, D. et al. Single-molecule spectroscopy of the temperature-induced collapse of unfolded proteins. Proceedings of the National Academy of Sciences 106, 20740-20745 (2009).

34 Shirts, M. R., Pitera, J. W., Swope, W. C. \& Pande, V. S. Extremely precise free energy calculations of amino acid side chain analogs: Comparison of common molecular mechanics force fields for proteins. The Journal of chemical physics 119, 5740-5761 (2003).

35 Petrov, D. \& Zagrovic, B. Are current atomistic force fields accurate enough to study proteins in crowded environments? PLoS Comput Biol 10, e1003638 (2014).

36 Nerenberg, P. S., Jo, B., So, C., Tripathy, A. \& Head-Gordon, T. Optimizing solute-water van der waals interactions to reproduce solvation free energies. The Journal of Physical Chemistry B 116, 4524-4534 (2012).

37 Best, R. B. \& Mittal, J. Protein simulations with an optimized water model: cooperative helix formation and temperature-induced unfolded state collapse. J Phys Chem B 114, 14916-14923, doi:10.1021/jp108618d (2010).

38 Abascal, J. L. \& Vega, C. A general purpose model for the condensed phases of water: TIP4P/2005. The Journal of chemical physics 123, 234505 (2005).

39 Zerze, G. H., Best, R. B. \& Mittal, J. Sequence- and Temperature-Dependent Properties of Unfolded and Disordered Proteins from Atomistic Simulations. The Journal of Physical Chemistry B 119, 14622-14630, doi:10.1021/acs.jpcb.5b08619 (2015). 
40 Rosenman, D. J., Wang, C. \& García, A. E. Characterization of A $\beta$ Monomers through the Convergence of Ensemble Properties among Simulations with Multiple Force Fields. The Journal of Physical Chemistry B 120, 259-277, doi:10.1021/acs.jpcb.5b09379 (2016).

41 Bhowmick, A. et al. Finding Our Way in the Dark Proteome. J Am Chem Soc 138, 97309742, doi:10.1021/jacs.6b06543 (2016).

42 Best, R. B., Zheng, W. W. \& Mittal, J. Balanced Protein-Water Interactions Improve Properties of Disordered Proteins and Non-Specific Protein Association. J Chem Theory Comput 10, 5113-5124 (2014).

43 Piana, S., Donchev, A. G., Robustelli, P. \& Shaw, D. E. Water Dispersion Interactions Strongly Influence Simulated Structural Properties of Disordered Protein States. The Journal of Physical Chemistry B 119, 5113-5123, doi:10.1021/jp508971m (2015).

44 Lindorff-Larsen, K. et al. Improved side-chain torsion potentials for the Amber ff99SB protein force field. Proteins: Structure, Function, and Bioinformatics 78, 1950-1958, doi:10.1002/prot.22711 (2010).

45 Henriques, J. \& Skepö, M. Molecular Dynamics Simulations of Intrinsically Disordered Proteins: On the Accuracy of the TIP4P-D Water Model and the Representativeness of Protein Disorder Models. J Chem Theory Comput 12, 3407-3415, doi:10.1021/acs.jctc.6b00429 (2016).

46 Rauscher, S. et al. Structural Ensembles of Intrinsically Disordered Proteins Depend Strongly on Force Field: A Comparison to Experiment. J Chem Theory Comput 11, 55135524, doi:10.1021/acs.jctc.5b00736 (2015).

47 Mercadante, D. et al. Kirkwood-Buff Approach Rescues Overcollapse of a Disordered Protein in Canonical Protein Force Fields. The Journal of Physical Chemistry B 119, 79757984, doi:10.1021/acs.jpcb.5b03440 (2015).

48 Levine, Z. A. et al. Surface force measurements and simulations of mussel-derived peptide adhesives on wet organic surfaces. Proceedings of the National Academy of Sciences 113, 4332-4337, doi:10.1073/pnas.1603065113 (2016).

49 Baker, C. M. \& Best, R. B. Insights into the binding of intrinsically disordered proteins from molecular dynamics simulation. Wiley Interdisciplinary Reviews: Computational Molecular Science 4, 182-198, doi:10.1002/wcms.1167 (2014).

50 Chen, J. H. Intrinsically Disordered p53 Extreme C-Terminus Binds to S100B(beta beta) through "Fly-Casting". J Am Chem Soc 131, 2088-2089 (2009).

51 De Sancho, D. \& Best, R. B. Modulation of an IDP binding mechanism and rates by helix propensity and non-native interactions: association of HIF1[small alpha] with CBP. Mol Biosyst 8, 256-267, doi:10.1039/C1MB05252G (2012).

52 Huang, Y. \& Liu, Z. Nonnative Interactions in Coupled Folding and Binding Processes of Intrinsically Disordered Proteins. . PLoS ONE 5, e15375, doi:doi:10.1371/journal.pone (2010).

53 Ganguly, D. et al. Electrostatically Accelerated Coupled Binding and Folding of Intrinsically Disordered Proteins. Journal of Molecular Biology 422, 674-684, doi:http://dx.doi.org/10.1016/i.jmb.2012.06.019 (2012).

54 Shoemaker, B. A., Portman, J. J. \& Wolynes, P. G. Speeding molecular recognition by using the folding funnel: the fly-casting mechanism. Proceedings of the National Academy of Sciences 97, 8868-8873 (2000). 
55 Higo, J. \& Umezawa, K. in Protein Conformational Dynamics (eds Ke-li Han, Xin Zhang, \& Ming-jun Yang) 331-351 (Springer International Publishing, 2014).

56 Huang, Y. \& Liu, Z. Kinetic Advantage of Intrinsically Disordered Proteins in Coupled Folding-Binding Process: A Critical Assessment of the "Fly-Casting" Mechanism. Journal of Molecular Biology 393, 1143-1159, doi:http://dx.doi.org/10.1016/j.jmb.2009.09.010 (2009).

57 Levy, Y., Onuchic, J. N. \& Wolynes, P. G. Fly-Casting in Protein-DNA Binding: Frustration between Protein Folding and Electrostatics Facilitates Target Recognition. J Am Chem Soc 129, 738-739, doi:10.1021/ja065531n (2007).

58 Morriss-Andrews, A. \& Shea, J. E. Computational Studies of Protein Aggregation: Methods and Applications. Annu Rev Phys Chem 66, 643-666, doi:10.1146/annurevphyschem-040513-103738 (2015).

59 Morriss-Andrews, A. \& Shea, J. E. Simulations of Protein Aggregation: Insights from Atomistic and Coarse-Grained Models. J Phys Chem Lett 5, 1899-1908, doi:10.1021/jz5006847 (2014).

60 Chiricotto, M., Melchionna, S., Derreumaux, P. \& Sterpone, F. Hydrodynamic effects on $\beta$-amyloid (16-22) peptide aggregation. The Journal of Chemical Physics 145, 035102, doi:doi:http://dx.doi.org/10.1063/1.4958323 (2016).

61 Latshaw, D. C., Cheon, M. \& Hall, C. K. Effects of Macromolecular Crowding on Amyloid Beta (16-22) Aggregation Using Coarse-Grained Simulations. The Journal of Physical Chemistry B 118, 13513-13526, doi:10.1021/jp508970q (2014).

62 Morriss-Andrews, A., Bellesia, G. \& Shea, J. E. beta-sheet propensity controls the kinetic pathways and morphologies of seeded peptide aggregation. J Chem Phys 137, doi:Artn 145104

10.1063/1.4755748 (2012).

63 Pellarin, R., Guarnera, E. \& Caflisch, A. Pathways and Intermediates of Amyloid Fibril Formation. Journal of Molecular Biology 374, 917-924, doi:http://dx.doi.org/10.1016/j.jmb.2007.09.090 (2007).

64 Carballo-Pacheco, M. \& Strodel, B. Advances in the Simulation of Protein Aggregation at the Atomistic Scale. The Journal of Physical Chemistry B 120, 2991-2999, doi:10.1021/acs.jpcb.6b00059 (2016).

65 Dominguez, L., Meredith, S. C., Straub, J. E. \& Thirumalai, D. Transmembrane Fragment Structures of Amyloid Precursor Protein Depend on Membrane Surface Curvature. J Am Chem Soc 136, 854-857, doi:10.1021/ja410958j (2014).

66 Cho, S. S., Reddy, G., Straub, J. E. \& Thirumalai, D. Entropic Stabilization of Proteins by TMAO. The Journal of Physical Chemistry B 115, 13401-13407, doi:10.1021/jp207289b (2011).

67 Baram, M., Atsmon-Raz, Y., Ma, B., Nussinov, R. \& Miller, Y. Amylin-A[small beta] oligomers at atomic resolution using molecular dynamics simulations: a link between Type 2 diabetes and Alzheimer's disease. Physical Chemistry Chemical Physics 18, 23302338, doi:10.1039/C5CP03338A (2016).

68 Schor, M., Mey, A. S. J. S., Noé, F. \& MacPhee, C. E. Shedding Light on the Dock-Lock Mechanism in Amyloid Fibril Growth Using Markov State Models. The Journal of Physical Chemistry Letters 6, 1076-1081, doi:10.1021/acs.jpclett.5b00330 (2015). 
69 O’Brien, E. P., Okamoto, Y., Straub, J. E., Brooks, B. R. \& Thirumalai, D. Thermodynamic Perspective on the Dock-Lock Growth Mechanism of Amyloid Fibrils. The Journal of Physical Chemistry B 113, 14421-14430, doi:10.1021/jp9050098 (2009).

70 Wu, C., Bowers, Michael T. \& Shea, J.-E. On the Origin of the Stronger Binding of PIB over Thioflavin T to Protofibrils of the Alzheimer Amyloid-<em>\&\#x3b2;</em> Peptide: A Molecular Dynamics Study. Biophysical Journal 100, 1316-1324, doi:10.1016/j.bpj.2011.01.058.

71 Feric, M. et al. Coexisting Liquid Phases Underlie Nucleolar Subcompartments. Cell 165, 1686-1697, doi:10.1016/j.cell.2016.04.047.

72 Pak, C. W. et al. Sequence Determinants of Intracellular Phase Separation by Complex Coacervation of a Disordered Protein. Mol Cell 63, 72-85, doi:10.1016/j.molcel.2016.05.042 (2016).

73 de Jong, B. H. G. \& Kruyt, H. R. Coacervation (partial miscibility in colloid systems)." Proc Koninklijke Nederlandse Akademie Wetenschappen 32: 849-856. Proc Koninklijke Nederlandse Akademie Wetenschappen 32, 849-856 (1929).

74 Overbeek, J. T. G. \& Voorn, M. J. Phase separation in polyelectrolyte solutions. Theory of complex coacervation. Journal of Cellular and Comparative Physiology 49, 7-26, doi:10.1002/jcp.1030490404 (1957).

75 Brangwynne, C. P., Tompa, P. \& Pappu, R. V. Polymer physics of intracellular phase transitions. Nat Phys 11, 899-904, doi:10.1038/nphys3532 (2015).

76 Fredrickson, G. H. Computational field theory of polymers: opportunities and challenges. Soft Matter 3, 1329-1334, doi:10.1039/B710604A (2007).

77 Lee, J., Popov, Y. O. \& Fredrickson, G. H. Complex coacervation: A field theoretic simulation study of polyelectrolyte complexation. J Chem Phys 128, doi:Artn 224908 10.1063/1.2936834 (2008).

78 Vitalis, A. \& Pappu, R. V. ABSINTH: A new continuum solvation model for simulations of polypeptides in aqueous solutions. Journal of Computational Chemistry 30, 673-699, doi:10.1002/jcc.21005 (2009).

79 Kang, H., Pincus, P. A., Hyeon, C. \& Thirumalai, D. Effects of Macromolecular Crowding on the Collapse of Biopolymers. Physical Review Letters 114, 068303 (2015).

80 Miller, Cayla M., Kim, Young C. \& Mittal, J. Protein Composition Determines the Effect of Crowding on the Properties of Disordered Proteins. Biophysical Journal 111, 28-37, doi:http://dx.doi.org/10.1016/j.bpj.2016.05.033 (2016).

81 Qin, S. \& Zhou, H.-X. Effects of Macromolecular Crowding on the Conformational Ensembles of Disordered Proteins. The Journal of Physical Chemistry Letters 4, 34293434, doi:10.1021/jz401817x (2013).

\section{Selected References}

** 11. An insightful computational study that classifies IDPs into different families based on the fraction of positively to negatively charged residues and their distribution in the polypeptide chain. 
* 16. An important computational advance for the structural ensemble determination of IDPs using a Bayesian approach.

** 42. The authors employ a novel approach to remedy deficiencies in IDP force fields by scaling the water-protein interactions. This strategy leads to better agreement with experiment in terms of IDP chain dimensions.

**43. The authors develop a novel water model that corrects for the fact that water dispersion interactions are underestimated in traditional physics-based water force fields. This new water model, Tip4P-D, when used in conjunction with protein force fields, led to more extended conformations for IDPs and to better agreement with experiment.

*46. The authors perform an extensive comparison of force field and water model combinations and show that IDP conformations can vary widely in terms of compactness and secondary structure content from force field to force field.

*72. One of the very first computational studies to address complex coacervation of biologically relevant systems.

*79. An insightful theoretical investigation into the effect of crowding on IDP conformations. 